\title{
Direct observation of ferroelectricity in a confined crystallite
}

\author{
P. Gupta, ${ }^{*}$ H. Jain, ${ }^{*}$ D.B. Williams, ${ }^{*}$ S.V. Kalinin, ${ }^{* *}$ J. Shin, ${ }^{* *}$ A.P. Baddorf $* *$ \\ *Department of Materials Science and Engineering, and Center for Optical Technologies, \\ Lehigh University, Bethlehem, PA 18015 \\ ** Condensed Matter Sciences Division, Oak Ridge National Laboratory, Oak Ridge, TN \\ 17831
}

Transparent ferroelectric glass-ceramics (TFGC) are being developed as an inexpensive and easy to fabricate material for-nonlinear optical applications. One of the most promising materials in the TFGC class is $\mathrm{LaBGeO}_{5}$ [1] (LBGO), which can be congruently precipitated in the bulk from the LBGO glass matrix through a two-step heat treatment of glass. While ferroelectric crystallites enable nonlinear optical activity, close matching between refractive indices of the crystallite and matrix ensures transparency to visible light. The nonlinear activity of LBGO TFGC has been observed through a second harmonic generation ( $\mathrm{SHG}$ ) experiment and it is found to increase with the number density of the crystallites. But, there are doubts about the origin of SHG due to possible clamping of the crystallites by the glass matrix, where clamping is defined as the suppression of the ferroelectric transformation of the crystallites in TFGC systems having the glass transition temperature $\left(\mathrm{T}_{\mathrm{g}}\right)$ higher than the ferroelectric transformation temperature $\left(\mathrm{T}_{\mathrm{c}}\right)$. Clamping has been reported for the lead titanate system by Lynch et al. [2] and Grossman et al. [3]. However, no direct observation of the clamping has been yet produced to convince that it is indeed effective to suppress the ferroelectric transformation. Thus, direct measurement of the ferroelectric properties of LBGO crystallites is performed. It is a step toward elucidating the role of ferroelectric crystallites in the nonlinear optical response for optimization of material morphology.

Here, we use piezoresponse force microscopy (PFM) to image ferroelectric properties of clamped LBGO crystallites on the nanoscale level. PFM [4] is a development of atomic force microscopy (AFM), in which a periodic voltage is applied to the conductive tip in contact with the sample surface. The mechanical response due to the converse piezoelectric effect in vertical and lateral directions causes tip bending and torsion proportional to the amplitude of the mechanical response of the sample. The amplitude and the phase of the mechanical response are collected using lock-in detection and are mapped simultaneously with the conventional topographic image.

The LBGO crystallites were precipitated from the congruent glass phase. The similarity of composition was confirmed from X-ray energy dispersive spectrometry (XEDS). PFM was performed on a crystallite of 20 micrometer size on the surface. Vertical and lateral piezoresponse images with their amplitude and phases imaged separately, were obtained. The vertical phase and amplitude images were combined to provide the vertical piezoresponse image (Fig. 1(a)). The lateral images were also combined to give lateral piezoresponse image (Fig. 1(b)). 
The PFM images clearly illustrate the strong electromechanical response of the crystallite, as compared to the glass matrix, confirming the existence of piezoelectricity in clamped crystallites. Considering the existence of ferrorlectricity in LBGO single crystals [5], crystallites can be labeled as ferroelectric. However, switching of domains through poling should be demonstrated in order to fully confirm ferroelectricity in crystallites. Also the piezoresponse images produced here are only good for qualitative interpretation and so there is still the possibility of weakening of the magnitude of the ferroelectric response due to clamping, which might have gone unnoticed. Thus, more careful experiments with simpler samples i.e. samples containing single crystals, for quantitative analysis are underway which will underscore the effect of the confining glass matrix on the magnitude of the piezoresponse of ferroelectric crystallites.

\section{References}

[1]P. Gupta, H. Jain, D. B. Williams, O. Kanert, R. Kuechler, J. Non-Cryst. Solids 349 (2004) 291-298.

[2] S. M. Lynch, J. E. Shelby, J. Am. Ceram. Soc., 67(6) (1984) 424-7..

[3] D. G. Grossman, J.O. Isard, J. Mat. Sc., 4(12) (1969) 1059-63.

[4] A. Gruverman, O. Auciello, H. Tokumoto, Annu. Rev. Mater. Sci., 28 (1998) 101-23.

[5] E.L. Belokeneva, W.I.F. David, J.M. Forsyth, K.S. Knight, J. Phys. Condens. Matter, 9 (1997) 3503.

[6] This work was supported by the Pennsylvania Department of Community and Economic Development through the Ben Franklin Technology Development Authority.
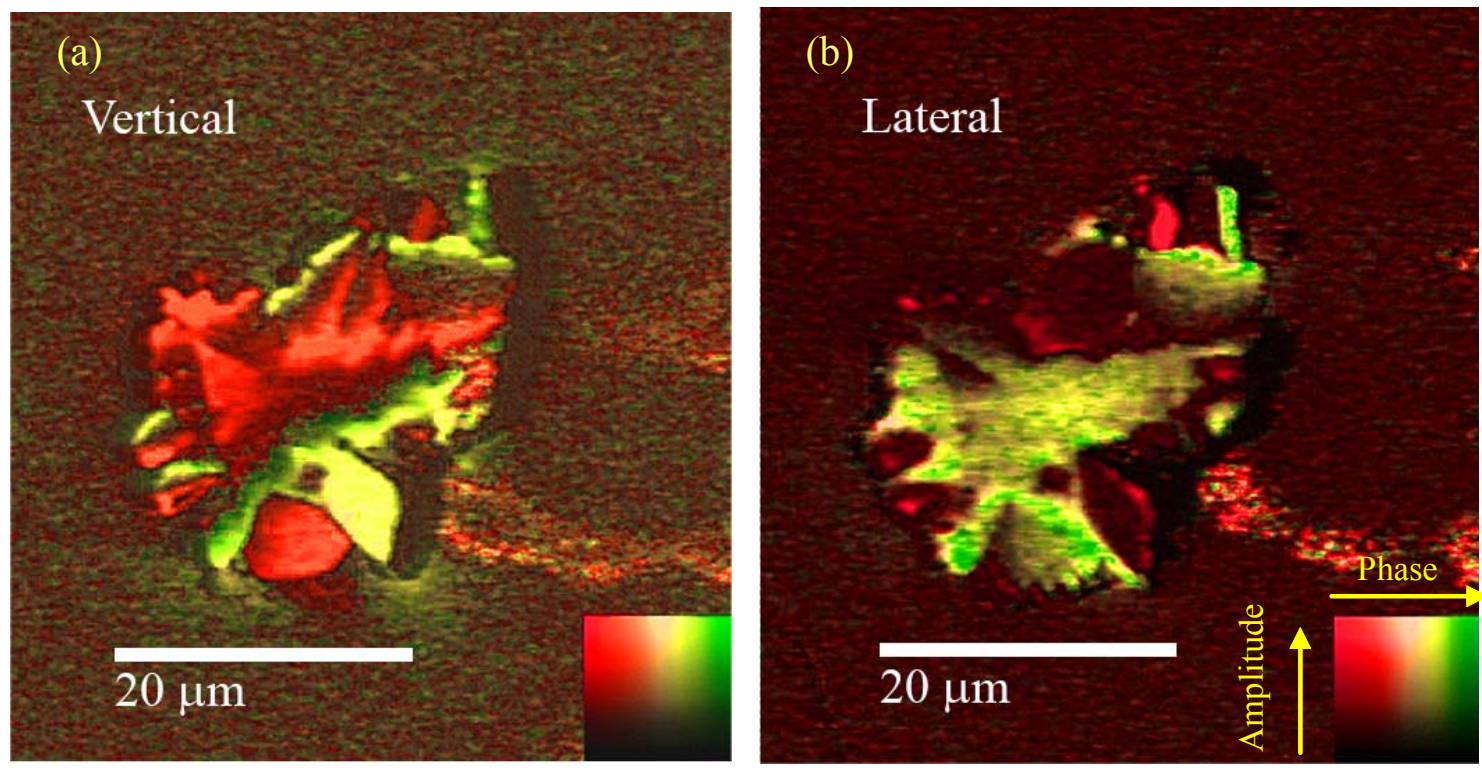

Fig.1. a) Vertical, and b) lateral piezoelectric response amplitude-phase images. The piezoelectric response in both the vertical and lateral directions has two components, amplitude and phase. Amplitude is encoded in the luminosity of colors and phase is encoded in the chromaticity of the colors. 